\title{
Focal HIFU therapy for anterior compared to posterior prostate cancer lesions
}

\author{
Philipp M. Huber ${ }^{1,2,5,7} \cdot$ Naveed Afzal $^{9} \cdot$ Manit Arya $^{5,7,8} \cdot$ Silvan Boxler ${ }^{1} \cdot$ Tim Dudderidge $^{10} \cdot$ Mark Emberton $^{4,5}$. \\ Stephanie Guillaumier ${ }^{4,5} \cdot$ Richard G. Hindley $^{11} \cdot$ Feargus Hosking-Jervis $^{6,7} \cdot$ Lucas Leemann $^{3} \cdot$ Henry Lewi $^{12}$. \\ Neil McCartan ${ }^{4,5} \cdot$ Caroline M. Moore $^{4,5} \cdot$ Raj Nigam $^{13} \cdot$ Chris Odgen $^{14} \cdot$ Raj Persad $^{15} \cdot$ Jaspal Virdi $^{8} \cdot$ Mathias Winkler $^{7}$. \\ Hashim U. Ahmed ${ }^{6,7}$
}

Received: 3 December 2019 / Accepted: 3 June 2020 / Published online: 7 July 2020

(c) The Author(s) 2020

\begin{abstract}
Objective To compare cancer control in anterior compared to posterior prostate cancer lesions treated with a focal HIFU therapy approach.

Materials and methods In a prospectively maintained national database, 598 patients underwent focal HIFU (Sonablate ${ }^{\circledR} 500$ ) (March/2007-November/2016). Follow-up occurred with 3-monthly clinic visits and PSA testing in the first year with PSA, every 6-12 months with mpMRI with biopsy for MRI-suspicion of recurrence. Treatment failure was any secondary treatment (ADT/chemotherapy, cryotherapy, EBRT, RRP, or re-HIFU), tumour recurrence with Gleason $\geq 3+4$ on prostate biopsy without further treatment or metastases/prostate cancer-related mortality. Cases with anterior cancer were compared to those with posterior disease.

Results 267 patients were analysed following eligibility criteria. 45 had an anterior focal-HIFU and 222 had a posterior focal-HIFU. Median age was 64 years and 66 years, respectively, with similar PSA level of $7.5 \mathrm{ng} / \mathrm{ml}$ and $6.92 \mathrm{ng} / \mathrm{ml} .84 \%$ and $82 \%$, respectively, had Gleason $3+4,16 \%$ in both groups had Gleason $4+3,0 \%$ and $2 \%$ had Gleason $4+4$. Prostate volume was similar ( $33 \mathrm{ml}$ vs. $36 \mathrm{ml}, p=0.315$ ); median number of positive cores in biopsies was different in anterior and posterior tumours ( 7 vs. $5, p=0.009$ ), while medium cancer core length, and maximal cancer percentage of core were comparable. $17 / 45$ (37.8\%) anterior focal-HIFU patients compared to $45 / 222(20.3 \%)$ posterior focal-HIFU patients required further treatment $(p=0.019)$.
\end{abstract}

Conclusion Treating anterior prostate cancer lesions with focal HIFU may be less effective compared to posterior tumours.

Keywords Prostate cancer $\cdot$ Focal therapy $\cdot$ High intensity focused ultrasound $\cdot$ Biochemical failure

Hashim U. Ahmed

hashim.ahmed@imperial.ac.uk

1 Department of Urology, University Hospital Inselspital Berne, Bern, Switzerland

2 Urologie St. Anna, Lucerne, Switzerland

3 Department of Political Science, University of Zurich, Zurich, Switzerland

4 Division of Surgery and Interventional Sciences, University College London, London, UK

5 Department of Urology, UCLH NHS Foundation Trust, London, UK

6 Imperial Prostate, Division of Surgery, Department of Surgery and Cancer, Faculty of Medicine, Imperial College London, Fulham Palace Road, London W6 8RF, UK

7 Imperial Urology, Charing Cross Hospital, Imperial College Healthcare NHS Trust, London, UK
8 Department of Urology, The Princess Alexandra Hospital NHS Trust, Harlow, UK

9 Department of Urology, Dorset County Hospital NHS Trust, Dorchester, Dorset, UK

10 Department of Urology, University Hospital Southampton NHS Trust, Southampton, UK

11 Department of Urology, Basingstoke Hospital, Hampshire Hospitals NHS Foundation Trust, Basingstoke, UK

12 Springfield Hospital, Chelmsford, Essex, UK

13 Department of Urology, Royal County Surrey Hospital NHS Trust, Guildford, Surrey, UK

14 Department of Academic Urology, The Royal Marsden Hospital NHS Foundation Trust, London, UK

15 Department of Urology, Southmead Hospital, North Bristol NHS Trust, Bristol, UK 


\section{Introduction}

Since high-intensity focused ultrasound (HIFU) was first started for the treatment of prostate cancer [1], treatment parameters have been refined from ablating the whole prostate gland to hemi-gland ablation, quadrant ablation and so-called ultra-focal ablation of cancerous areas [2]. Less than whole-gland approaches seem to confer reassuring medium-term cancer control with reduced side-effects and complications [3], provided patients are staged and selected appropriately [4].

The energy source for HIFU is usually placed in the patient's rectum, with ultrasound waves having to traverse a number of tissue planes. There can be loss of energy by passing multiple tissue planes and large volumes of tissue between source and prostate tumour. There has been some concern as to whether HIFU is able to confer similar cancer control rates for treating anterior prostate tumours. Indeed, the reported local intraprostatic swelling and prostate shift during the treatment [5] may have a higher impact on anterior parts of the prostate, with the anterior border of the lesion drifting away from the energy. We aimed to compare cancer control in anterior compared to posterior prostate cancer lesions treated with a focal HIFU therapy approach.

\section{Methods}

The UCLH Joint Research Office granted institutional review board exemption. Between March/2007 and November/2016, 598 consecutive patients underwent primary focal HIFU (focal lesion ablation or quadrant or hemiablation) for non-metastatic prostate cancer using the Sonablate ${ }^{\circledR} 500$ device (Sonacare Inc., USA) within nine centres. Hemiablations were excluded from this analysis. Prior to consensus articles which streamlined eligibility criteria for focal therapy in prostate cancer, it was not known nor agreed which cases may benefit most. As a result, focal HIFU treatment was initially offered to patients diagnosed with non-metastatic prostate cancer with Gleason 6 through 9, stage T1c-T3bN0M0 and PSA of $\leq 20 \mathrm{ng} / \mathrm{ml}$. Gleason 6 required a minimum of $3 \mathrm{~mm}$ of disease. Over the last 5 years, the UK Focal Therapy Users Group predominantly treats men with stage T1cT2cN0M0, Gleason 7 and PSA $\leq 20 \mathrm{ng} / \mathrm{ml}$. For this analysis, only patients with Gleason $\geq 7$ were included since positive recurrence was defined as Gleason $\geq 7$ as described below. Furthermore, for all cases analysed prostate size in the group with tumour located in posterior area of the gland was limited to the maximum of prostate size of the comparator group. 267 patients were eligible for analysis in this study as they had more than 3 months follow-up and information on tumour location. Disease was localised using mpMRI, combined with targeted and systematic biopsies, or transperineal mapping biopsies. Cases were separated into anterior or posterior groups according to tumour location in mpMRI where each prostate gland was arithmetically divided in half in anterior-posterior dimensions.

Treatments included in this analysis were delivered in a focal lesion ablation or quadrant fashion depending on the gland volume as well as tumour volume and location. Index lesion ablation alone was conducted in patients with multifocal disease provided untreated areas harboured no more than $3 \mathrm{~mm}$ of Gleason 6 on systematic or template mapping biopsies. All men were advised to undergo 3-6 monthly serum PSA testing. An mpMRI was routinely performed regardless of PSA changes at 1 year and approximately 1-2 yearly thereafter. Two rises in PSA after the nadir level was achieved, without predefining the level of rise, was investigated with a prostate biopsy, or mpMRI followed by biopsy if the mpMRI was suspicious. We have previously reported on the high negative predictive value of mpMRI in the post-focal HIFU setting for clinically significant prostate cancer [6]. Clinically significant cancer on biopsy of untreated areas was defined as 'out-of-field' progression.

Treatment failure was defined by any secondary treatment (ADT/chemotherapy, cryotherapy, EBRT, RRP, or re-HIFU), metastasis from prostate cancer without further treatment, tumour recurrence with Gleason score $\geq 7$ proved by prostate biopsy without further treatment, or death from prostate cancer, respectively.

\section{Statistical analysis}

Variables with skewed distribution are reported as median (interquartile ranges, IQR). Categorical variables are reported as absolute numbers with percentages. Significance levels for median values were calculated with Mood's Median Test, and treatment results with Fisher's Exact Binomial Test. Multivariate analysis was performed with logit models using the following factors: age, PSA level pre-treatment, and prostate volume were used as continuous parameters, whereas tumour location (anterior vs. posterior), clinical T stage ( $<\mathrm{cT} 3$ vs. $\geq$ cT3), and Gleason score $(<4+3$ vs. $\geq 4+3)$ were categorised. Analyses were performed using the $\mathrm{R}$ language environment for statistical computing. $p<0.05$ was considered as statistically significant. 


\section{Results}

\section{Baseline demographics}

From a total of 598 focal primary HIFU cases, and applying eligibility criteria 267 patients were analysed, divided into a group of 45 cases with an anterior, and a group of 222 cases with a posterior located prostate tumour treatment (Table 1). Since prostate volume in posterior tumour group was limited to the maximum of prostate volume in anterior tumour group there was no significant difference. In the anterior group, no Gleason $4+4$ was treated while in the posterior group $2 \%$ of cases were Gleason $4+4$. The number of positive cores in biopsies pre-treatment was significantly higher in anterior tumour group compared to posterior tumour location (median 7 vs. $5, p=0.009$ ).

\section{Treatment outcome}

The two groups showed statistically significant different failure rates. In anteriorly located tumours failure occurred in $17 / 45$ cases $(37.8 \%)$, whereas in posteriorly located tumours $45 / 222$ cases $(20.3 \%)$ had a treatment failure $(p=0.019)$. There were no differences in time to treatment failure or follow-up without failure (Table 2). In multivariate analysis using logit models anterior tumour location and $\geq \mathrm{cT} 3$ stage resulted as a significant predictor for treatment outcome (Table 3).

\section{Discussion}

In summary, we have shown that failure following focal HIFU is related to the position of the cancer in the anterior-posterior plane. Prior to discussing the clinical implications of our study, there are some limitations that require mentioning. First, it is a retrospective analysis of a national database with heterogeneity in case-mix. Second, there was not an underlying protocol, so we could not mandate biopsy in all men, particularly if the PSA was stable. Third, we lack long term evaluation of metastases and mortality and therefore used a composite endpoint [7]. Last, we were not able to evaluate whether apical versus mid-gland or base locations had impact on the failure rates.

Although focal treatment modalities have demonstrated their ability in treating prostate cancer [8], there are some technical limitations in HIFU. The probe used in our study (Sonablate500) has two pre-defined focal energy points at $4 \mathrm{~cm}$ and $3 \mathrm{~cm}$ lengths, there is an inherent limitation for glands which are large or have an AP length of much greater than $3.5 \mathrm{~cm}[9,10]$. What we have shown is that

Table 1 Baseline demographics

\begin{tabular}{|c|c|c|c|c|c|}
\hline \multirow[t]{2}{*}{ Determinant } & \multicolumn{2}{|l|}{ Anterior tumour } & \multicolumn{2}{|l|}{ Posterior tumour } & \multirow[t]{2}{*}{$p$ value } \\
\hline & Median/mean/N & $\mathrm{IQR} / \%$ & Median/mean $/ N$ & $\mathrm{IQR} / \%$ & \\
\hline Age at treatment (in years), median, (IQR) & 64 & $59-68$ & 66 & $60-71$ & 0.531 \\
\hline ADT pre treatment, $N,(\%)$ & 5 & $11 \%$ & 24 & $11 \%$ & 0.970 \\
\hline PSA pre treatment (in $\mathrm{ng} / \mathrm{ml})$, median, (IQR) & 7.53 & $5.22-10.77$ & 6.92 & $5.05-9.64$ & 0.496 \\
\hline Prostate volume (MRI, in ml), mean, (range) & 33 & $28-40$ & 36 & $28-47$ & 0.315 \\
\hline \multicolumn{6}{|l|}{ Biopsy results pre treatment, median, (IQR) } \\
\hline No. positive cores & 7 & $3-13$ & 5 & $4-9$ & 0.009 \\
\hline$M C C L($ in $\mathrm{mm})$ & 6.5 & $5-8.75$ & 6 & $4-8$ & 0.597 \\
\hline Max. cancer percentage of core $(\%)$ & 50 & $40-76$ & 60 & $39-75$ & 0.507 \\
\hline Gleason score pre treatment, median & $3+4$ & & $3+4$ & & \\
\hline $3+4$ & 38 & $84 \%$ & 181 & $82 \%$ & 0.832 \\
\hline $4+3$ & 7 & $16 \%$ & 36 & $16 \%$ & 1.000 \\
\hline $4+4$ & 0 & $0 \%$ & 5 & $2 \%$ & 0.593 \\
\hline T-stage pre treatment, median, $N,(\%)$ & $\mathrm{T} 2$ & & $\mathrm{~T} 2$ & & \\
\hline$T 1 c$ & 5 & $11 \%$ & 10 & $4 \%$ & 0.145 \\
\hline$T 2 a$ & 3 & $7 \%$ & 15 & $7 \%$ & 1.000 \\
\hline$T 2 b$ & 27 & $60 \%$ & 117 & $53 \%$ & 0.415 \\
\hline$T 2 c$ & 6 & $13 \%$ & 50 & $22 \%$ & 0.228 \\
\hline$T 3 a$ & 4 & $9 \%$ & 28 & $13 \%$ & 0.619 \\
\hline$T 3 b$ & 0 & $0 \%$ & 2 & $1 \%$ & 1.000 \\
\hline
\end{tabular}

$N$ number, $I Q R$ inter quartile range, $A D T$ androgen deprivation therapy, $M R I$ magnetic resonance imaging, $m l$ millilitres, $M C C L$ maximum cancer core length, $\mathrm{cm}$ centimetre 
Table 2 Treatment outcomes

\begin{tabular}{|c|c|c|c|c|c|}
\hline \multirow[t]{2}{*}{ Determinant } & \multicolumn{2}{|l|}{ Anterior tumour } & \multicolumn{2}{|c|}{ Posterior tumour } & \multirow[t]{2}{*}{$p$ value } \\
\hline & Median/mean $/ N$ & IQR/range/\% & $\begin{array}{l}\text { Median/ } \\
\text { mean/N }\end{array}$ & IQR/range/\% & \\
\hline Total patients, $N$ & 45 & & 222 & & \\
\hline Recurrences in treated area, $N,(\%)$ & 17 & $37.8 \%$ & 45 & $20.3 \%$ & 0.019 \\
\hline \multicolumn{6}{|l|}{ Proof of recurrence by } \\
\hline Biopsy, $N$, (\% of recurrences) & 8 & $47 \%$ & 22 & $49 \%$ & 0.930 \\
\hline mpMRI, $N$, (\% of recurrences) & 9 & $53 \%$ & 23 & $51 \%$ & 1.074 \\
\hline Time to recurrence (mth), mean, (range) & 28.5 & $22-41$ & 21 & $18-36$ & 0.274 \\
\hline Follow-up without recurrence (mth), mean, (range) & 29 & $15.5-46$ & 19 & $11-41$ & 0.030 \\
\hline \multicolumn{6}{|l|}{ Secondary treatment modality } \\
\hline Secondary HIFU, $N$, (\% of recurrences) & 9 & $53 \%$ & 39 & $87 \%$ & \\
\hline Cryoablation, $N$, (\% of recurrences) & 6 & $35 \%$ & 0 & $0 \%$ & \\
\hline Radical prostatectomy, $N$, (\% of recurrences) & 1 & $6 \%$ & 5 & $11 \%$ & \\
\hline Radiotherapy, $N$, (\% of recurrences) & 1 & $6 \%$ & 1 & $2 \%$ & \\
\hline
\end{tabular}

$N$ number, $I Q R$ inter quartile range, $m p M R I$ multiparametric MRI, $m t h$ month, $A D T$ androgen deprivation therapy

Table 3 Multivariate analysis: predictors for treatment failure

\begin{tabular}{lllr}
\hline Determinant & OR & IQR & $p$ value \\
\hline Age at treatment & 0.973 & $0.93-1.01$ & 0.192 \\
PSA pre-treatment & 1.054 & $0.98-1.13$ & 0.152 \\
Prostate volume & 0.992 & $0.97-1.02$ & 0.526 \\
Anterior tumour location & 0.304 & $0.14-0.64$ & 0.002 \\
Clinical T stage $\geq$ cT3 & 3.831 & $2.03-7.39$ & $<0.001$ \\
Gleason score $\geq 4+3$ & 1.754 & $0.80-3.75$ & 0.152 \\
\hline
\end{tabular}

$O R$ odds ratio, $I Q R$ inter quartile range

even when the probe can reach the lesion there is a relevant difference in successful treating of cancers localised more anteriorly compared to those closer to the rectal probe. There still seems to be an issue with ablative efficacy likely related to loss of energy as the pulse traverses multiple planes of tissue. With the high resolution and precision of a mpMRI for outlining prostate cancer boarders an accurate planning instrument is available [11]. The distance between rectal wall and the most anterior tumour border can be defined precisely, and unsuitable cases for HIFU with a tumour location beyond the maximal focal point have to be excluded. In our practice, we now use interstitial needle based ablative therapies $[12,13]$.

When planning a HIFU treatment for anterior prostate tumours one should bear in mind the intraprostatic changes, which are produced by heating energy through ablation. There is an intraprostatic swelling phenomenon that occurs as well as shifting of the prostate, demonstrated for wholegland HIFU treatments [5], and even when a smaller area is treated focally, a certain shift of the targeted area away from the focal point has to be kept in mind.
Unlike in other focal ablative technologies-like cryotherapy, irreversible electroporation or laser approacheswhere ablative energy can be delivered directly into a desired intraprostatic area, the HIFU energy source distributes its energy from an extra-prostatic point-source. Therefore, there is a higher loss of ablative energy in anterior located tumours, when more tissue needs to be crossed to reach the region of interest. For anterior areas, there is always more energy absorbed by posterior prostatic parenchyma than for posterior areas [14]. Whilst overall rates of cancer control are acceptable in the medium term in men with clinically significant prostate cancer [15], in this retrospective analysis a significantly higher recurrence rate for anterior located prostate cancers compared to posterior tumours is demonstrated. When comparing the underlying baseline demographics, there is a significant higher number of positive cores in the anterior tumour group. This difference must be a result of different number of total biopsy cores taken, since there was no protocol used how many cores have to be taken from suspicious lesions.

\section{Conclusion}

Treating anterior prostate cancer lesions with focal HIFU may be less effective compared to posterior tumours.

Author contributions PH, HUA, SG and NM were responsible for data collection, analysis of the data. HUA and $\mathrm{PH}$ were responsible for production of the first draft. PH and HUA completed the data analysis. All authors were involved in data collection, manuscript preparation/ drafting and approval of the final draft. HUA had full access to all of the data in the study and takes responsibility for the integrity of the data 
and the accuracy of the data analysis. HUA is guarantor of the study. PMH: Protocol/project development, Data collection or management, Data analysis, Manuscript writing/editing. NA: Data collection or management. MA: Data collection or management. SB: Protocol/project development, Data analysis. TD: Data collection or management. ME: Data collection or management. SG: Data collection or management. RGH: Data collection or management. FH-J: Data collection or management. LL: Data analysis. HL: Data collection or management. NM: Data collection or management. CMM: Data collection or management. RN: Data collection or management. CO: Data collection or management. RP: Data collection or management. JV: Data collection or management. MW: Data collection or management. HUA: Protocol/ project development, Data collection or management, Data analysis, Manuscript writing/editing.

Funding None of the funding sources had any role or input into the design and conduct of the study; collection, management, analysis, and interpretation of the data; and preparation, review, or approval of the manuscript.

\section{Compliance with ethical standards}

Conflict of interest Ahmed received funding from the Medical Research Council (UK) for this study. Ahmed and Emberton received an unrestricted grant from Sonacare Inc. for this work. Ahmed's research is supported by core funding from the United Kingdom's National Institute of Health Research (NIHR) Imperial Biomedical Research Centre. Ahmed currently receives funding from the Wellcome Trust, Medical Research Council (UK), Prostate Cancer UK, Cancer Research UK, The BMA Foundation, The Urology Foundation, The Imperial Health Charity, Sonacare Inc., Trod Medical and Sophiris Biocorp for trials and studies in prostate cancer. Ahmed is a paid medical consultant for Sophiris Biocorp, Sonacare Inc. and BTG/Galil. Mark Emberton receives research support from the United Kingdoms's National Institute of Health Research (NIHR) UCLH/UCL Biomedical Research Institute. He is an NIHR Senior Investigator. Emberton receives funding from NIHR-i4i, Medical Research Council (UK), Cancer Research UK, The Jon Moulton Foundation, Sonacare Inc., Trod Medical, and Sophiris Biocorp for trials in prostate cancer. Emberton is a medical consultant to Sonacare Inc., Sophiris Biocorp, Steba Biotech, Exact Imaging and Profound Medical. Moore receives funding from the National Institute for Health Research, The European Association of Urology Research Foundation, Prostate Cancer UK, Movember and the Cancer Vaccine Institute, for clinical prostate cancer research. She has received advisory board fees for Genomic Health. Ahmed, Emberton, Hindley, Moore, Boxler and Arya are all proctors for HIFU and are paid for training other surgeons in this procedure. Emberton, Freeman and Hindley have loan notes/stock options in Nuada Medical Ltd (UK). Winkler receives a travel grant and a loan of device from Zicom Biobot. None of the other authors have anything to declare.

Informed consent All cases had to be prospectively and consecutively entered into an academic registry according to UK national guidelines. Therapy options were discussed in a multidisciplinary meeting. Before treatment information on the advantages and disadvantages of the procedure had to be discussed with all patients. Written consent on therapy modality were given by all cases.

Open Access This article is licensed under a Creative Commons Attribution 4.0 International License, which permits use, sharing, adaptation, distribution and reproduction in any medium or format, as long as you give appropriate credit to the original author(s) and the source, provide a link to the Creative Commons licence, and indicate if changes were made. The images or other third party material in this article are included in the article's Creative Commons licence, unless indicated otherwise in a credit line to the material. If material is not included in the article's Creative Commons licence and your intended use is not permitted by statutory regulation or exceeds the permitted use, you will need to obtain permission directly from the copyright holder. To view a copy of this licence, visit http://creativecommons.org/licenses/by/4.0/.

\section{References}

1. Valerio M, Ahmed HU, Emberton M et al (2014) The role of focal therapy in the management of localised prostate cancer: a systematic review. Eur Urol 66(4):732-751

2. Ahmed HU, Hindley RG, Dickinson L et al (2012) Focal therapy for localised unifocal and multifocal prostate cancer: a prospective development study. Lancet Oncol 13(6):622-632

3. Ahmed HU, Dickinson L, Charman S et al (2015) Focal ablation targeted to the index lesion in multifocal localised prostate cancer: a prospective development study. Eur Urol 68(6):927-936

4. Donaldson IA, Alonzi R, Barratt D et al (2015) Focal therapy: patients, interventions, and outcomes-a report from a consensus meeting. Eur Urol 67(4):771-777

5. Shoji S et al (2013) Prostate swelling and shift during high intensity focused ultrasound: implication for targeted focal therapy. J Urol 190(4):1224-1232

6. Dickinson L et al (2017) Prostate-specific antigen vs. magnetic resonance imaging parameters for assessing oncological outcomes after high intensity-focused ultrasound focal therapy for localized prostate cancer. Urol Oncol 35(1):30.e9-30.e15

7. Peters $\mathrm{M}$ et al (2018) Development and internal validation of prediction models for biochemical failure and composite failure after focal salvage high intensity focused ultrasound for local radiorecurrent prostate cancer: presentation of risk scores for individual patient prognoses. Urol Oncol 36(1):13.e1-13.e10

8. Sivaraman A et al (2016) Focal therapy for prostate cancer: an "À la Carte" approach. Eur Urol 2015(69):973-975

9. Gelet A et al (1993) Prostatic tissue destruction by high-intensity focused ultrasound: experimentation on canine prostate. $\mathrm{J}$ Endourol 7(3):249-253

10. Rouvière $\mathrm{O}$ (2012) Imaging techniques for local recurrence of prostate cancer: for whom, why and how? Diagn Interv Imaging 93(4):279-290

11. Ahmed HU, El-Shater Bosaily A, Brown LC, PROMIS study group et al (2017) Diagnostic accuracy of multi-parametric MRI and TRUS biopsy in prostate cancer (PROMIS): a paired validating confirmatory study. Lancet 389(10071):815-822

12. Giganti F, Stabile A, Giona S et al (2019) Prostate cancer treated with irreversible electroporation: MRI-based volumetric analysis and oncological outcome. Magn Reson Imaging 58:143-147

13. Shah TT, Peters M, Eldred-Evans D et al (2019) Early-mediumterm outcomes of primary focal cryotherapy to treat nonmetastatic clinically significant prostate cancer from a prospective multicentre registry. Eur Urol 76(1):98-105

14. Haar GT et al (2007) High intensity focused ultrasound: physical principles and devices. Int J Hyperth 23(2):89-104

15. Guillaumier S, Peters M, Arya M et al (2018) A multicentre study of 5-year outcomes following focal therapy in treating clinically significant nonmetastatic prostate cancer. Eur Urol 74(4):422-429

Publisher's Note Springer Nature remains neutral with regard to jurisdictional claims in published maps and institutional affiliations. 